\title{
Tapered fiber coated with hydroxyethyl cellulose/polyvinylidene fluoride composite for relative humidity sensor
}

\author{
A. Lokman ${ }^{\mathrm{a}}$, H. Arof ${ }^{\mathrm{a}, *}$, S.W. Harun ${ }^{\mathrm{b}}$ \\ a Department of Electrical Engineering, Faculty of Engineering, University of Malaya, 50603, Kuala Lumpur, Malaysia \\ b Photonics Research Center, University of Malaya, 50603, Kuala Lumpur, Malaysia
}

\section{A R T I C L E I N F O}

Article history:

Received 26 December 2014

Received in revised form 10 February 2015

Accepted 10 February 2015

Available online 18 February 2015

\section{Keywords:}

Hydroxyethyl Cellulose/Polyvinylidene

Fluoride

Fiber Sensor

Inline MZI

Inline Mzl

humidity sensor

\section{A B S T R A C T}

A new evanescent wave based sensor is proposed and demonstrated using a silica fiber interferometer coated with Hydroxyethyl Cellulose/Polyvinylidene Fluoride (HEC/PVDF) composite. The performance of the sensor is investigated for two different types of interferometer structure: inline Mach Zehnder Interferometer (MZI) with dumbell structure and non-adiabatic etched fiber. The measurement is based on interferometric technique where the transmission spectrum of the reflected light is investigated for changes in relative humidity. For instance, the resonant dip wavelength for MZI dumbbell shape increases from 1555.76 to $1556.34 \mathrm{~nm}$ as the RH increases from 10 to $80 \%$. While, for etched SMF the resonant dip wavelength increases from 1554.58 to $1554.85 \mathrm{~nm}$ as the RH increases from 10 to $80 \%$. Both sensors demonstrated a linear shift especially within a range from 20 to $45 \%$. It is found that the MZI-based sensor has a sensitivity of $0.0123 \mathrm{~nm} / \%$ with a linearity of $99.88 \%$ and limit of detection of $0.44 \%$. On the other hand, the etched SMF structure also shows change in the resonant wavelength with the increase in RH. The tapered fiber based sensor has a sensitivity of $0.0074 \mathrm{~nm} / \%$ with linearity of $98.85 \%$ and limit of detection of $0.65 \%$. The lower limit of detection for dumbbell structure shows that the system is more efficient than etched SMF structure. The proposed sensor has a high potential for RH measurement as it has easy to fabricate, low fabrication cost, and compact size.

(c) 2015 Elsevier B.V. All rights reserved.

\section{Introduction}

Humidity monitoring is crucial in numerous industries like mining, chemical and biomedical plants as it may affect product quality and workers' health. Humidity is also monitored in big structures such as bridges or planes to control possible risks of corrosion [1]. Therefore relative humidity measurement has been extensively studied and a great variety of sensors, including capacitive, resistive, thermal conductivity and optical humidity sensors have been developed in last few decades. So far, the humidity sensor marke is monopolized by electronic sensors that enjoy well established fabrication technology. However, the field of optical fiber sensors has grown enormously and there exist niche applications where optical fiber humidity sensors can advantageously compete with their electronics counterparts.

Fiber optic humidity sensors (FOHS) use optical fiber technology to guide a light signal which is modulated by the fiber core and the surrounding medium and then collected back by

\footnotetext{
* Corresponding author.

E-mail address: ahamzah@um.edu.my (H. Arof).

http://dx.doi.org/10.1016/j.sna.2015.02.015

0924-4247/@ 2015 Elsevier B.V. All rights reserved.
}

a detector in conditioned and processed form. Since humidity affects the refractive index of the surrounding medium, changes in relative humidity alters the transmitted or reflected light that interacts with the medium. It is possible to transmit large amount of sensor data over a long distance due to the low attenuation and large wideband of optical fiber. In addition, the use of several interrogating techniques enable the application of distributed humidity sensor configurations [2]. There are also a number of applications where possible electrical hazards in volatile environment or electromagnetic interference in the surrounding may hinder the use of electronic humidity sensors. Furthermore, small dimension and simple geometry enable optical fiber sensors to be constructed and packaged into light-weight systems or embedded in polymer material ergonomically. Finally, when designed to operate at a high resolution, optical fiber sensors normally show a higher sensitivity and dynamic range than conventional sensors.

A number of different approaches have been used in the fabrication of optical fiber humidity sensors [3-7]. Most of these sensors are based on the interaction of evanescent wave of a tapered fiber with the surrounding. For instance, Muto et al. [8] demonstrated humidity sensors based on reversible absorption of water $\left(\mathrm{H}_{2} \mathrm{O}\right)$ from the ambient atmosphere into a porous thinfilm 


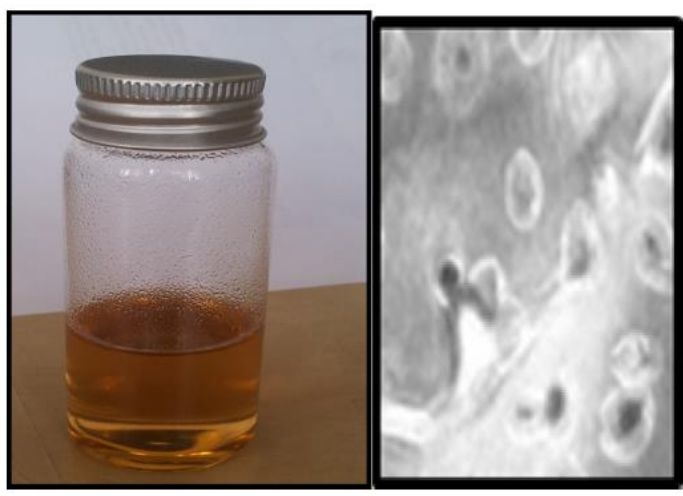

Fig. 1. The polymer composite solution and its microscopic image.

interferometer that sits on a tapered fiber. The water absorbed by the thin film changes its refractive index and subsequently transforms the lossy fiber into a light guide. In another work, Corres et al. [9] demonstrated a similar humidity sensor based on nanostructured films, which were deposited onto tapered fibers using the ionic self-assembled monolayer (ISAM) deposition technique $[10,11]$. The advantage of this scheme is that the sensing element can be fabricated using inexpensive components and a variety of coating materials can be deposited onto the tapered fiber. In our earlier work, a relative humidity $(\mathrm{RH})$ sensor is proposed and demonstrated based on intensity modulation in a tapered plastic optical fiber (POF). The tapered POF is coated with a polymer blend of hydroxyethylcellulose/polyvinylidenefluoride (HEC/PVDF) composite. Changes in relative humidity alter the physical and optical properties of the coating thereby influencing the evanescent waves that travels within the coating [12]. In this paper, a new evanescent wave based sensor is proposed and demonstrated using an interferometer silica fiber coated with HEC/PVDF composite. The performance of the sensor is investigated for two different types of interferometer structure: inline Mach Zehnder Interferometer (MZI) with dumbell structure and nonadiabatic tapered fiber. The measurement is based on interferometric technique where the transmission spectrum of the reflected light is investigated with respect to changes in relative humidity.

\section{Experimental arrangement}

Preliminary research works have reported numerous sensing materials, such as polyimide, crystal violet, porous silica xerogel [13], agarose, and a variety of methyl polymers that can be used to coat tapered fiber for humidity measurement. Here, we choose a combination of hydroxyethyl cellulose (HEC) and polyvinylidene fluoride (PVDF), as the sensing materials since they are relatively common and inexpensive. For the preparation of HEC/PVDF mixture, $1 \mathrm{~g}$ of PVDF powder $\left(M_{\mathrm{w}}=275,000\right)$ is dissolved in $120 \mathrm{ml}$ dimethyl sulfoxide (DMSO) and $100 \mathrm{ml}$ distilled water at $90^{\circ} \mathrm{C}$ in water bath. Then the solution of PVDF is cooled down to room temperature and $4 \mathrm{~g}$ of hydroxyethyl cellulose (HEC) is added to the solution. The mixed solution is continuously stirred at room temperature for about $10 \mathrm{~h}$ in order to generate a three-dimensional structure of the mesh gel (hydrogel). DMSO is used in the preparation of the HEC/PVDF as solvent since HEC is only soluble in water while PVDF is insoluble in water. Fig. 1 shows the sample of the polymer composite solution, which is obtained by blending HEC and PVDF and its microscopic image [12].

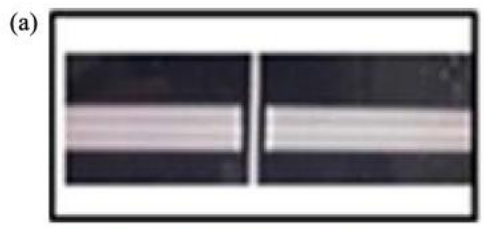

(b)

(c)
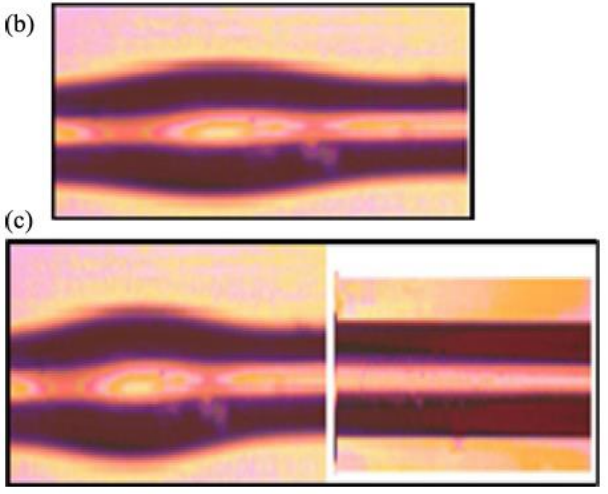

Fig. 2. (a) The first step in fabricating the MZI structure. (b) The first bulge of MZI structure. (c) The second bulge is formed in a similar fashion.

The inline MZI is then fabricated using a standard silica singlemode fiber (SMF), fiber striper, a cleaver and a fusion splicer machine. The SMF used has a core and cladding diameters of $8.3 \mu \mathrm{m}$ and $125 \mu \mathrm{m}$, respectively. The first step in fabricating the MZI structure is to strip and cleave an SMF into two sections, each with a flat and smooth end-surface, as illustrated in Fig. 2(a). Then the end facets of the two sections are matched and fused using the manua "arc "function of the splicer machine. While fusing the two sections of the fiber, the "arc" function is used so that extra pressure is exerted from both sides to form the first bulge at the joint as shown in Fig. 2(b). Once the first bulge is formed, the jointed fiber is then cleaved once again at $1 \mathrm{~mm}$ away from the center of the first bulge as depicted in Fig. 2(c). Then the second bulge is formed in a similar fashion. It should be noted that the fusion splicer softens the ends of the two fibers with heat before pulling them together to join them at the center. When the "arc" function is used repeatedly, more material is lumped at the center, thus forming a bulge, while the neck of the bulge gets thinner. When the second bulge is made, it is a bit smaller in size than the first one as less material is available from the waist area between the bulges since it has been used to form the first bulge. The waist area also becomes thinner as it gets tapered even more. The completed dumbbell structure is shown in Fig. 3 where the diameters of the first and second bulges are approximately around $198 \mu \mathrm{m}$ and $196 \mu \mathrm{m}$, respectively. Next, the prepared HEC/PVDF composite solution is slowly dropped onto the sensor using a syringe and left to dry for $48 \mathrm{~h}$.

Fig. 4 shows the experimental setup for the proposed sensor that detects changes in relative humidity using the fabricated inline dumbbell shaped MZI coated with HEC/PVDF composite. The input signal from the amplified spontaneous emission (ASE) of Erbium Doped Fiber Amplifier operating in $1550 \mathrm{~nm}$ region is launched into a sensor probe via a $3 \mathrm{~dB}$ coupler. The reflected signal from the sensor is then routed into an optical spectrum analyzer (OSA) with a resolution of $0.05 \mathrm{~nm}$ through the same coupler. The sensor probe is placed in a sealed chamber with a dish filled with saturated salt solution. Exposing the HEC/PVDF composite to the $\mathrm{RH}$ changes inside the chamber produces variations in the transmission 


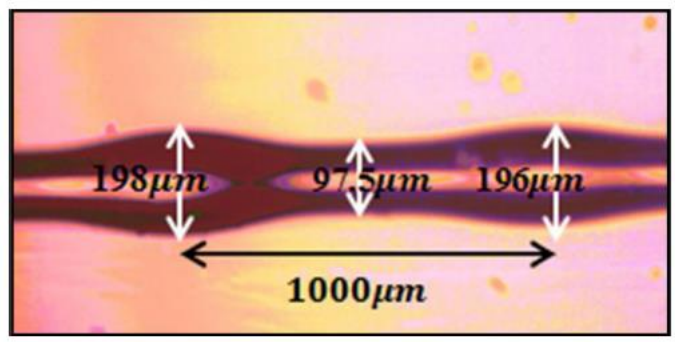

Fig. 3. The experimental view of the proposed dumbbell shaped MZI.

spectrum. In the experiment, the performance of the proposed sensor was investigated for various changes in relative humidity ranging from $10 \%$ to $80 \%$ using a humidity meter with a data logging capability.

Then, the same experiment was repeated by using a nonadiabatic tapered silica fiber coated with the HEC/PVDF composite as a probe for comparison purpose. A tapered fiber was prepare from a standard SMF using a chemical etching technique. The jacket of a small section of the fiber (about several $\mathrm{cm}$ in length) was removed prior to the etching process. Then, the unjacketed fiber is immersed into a hydrofluoric acid solution (47\%) for about $30 \mathrm{~min}$ to reduce the cladding diameter from 125 to $50 \mu \mathrm{m}$. The etched SMF was cleansed using deionized water before the prepared HEC/PVDF composite solution was slowly dropped onto it (using syringe) and left to dry for 48 hours

\section{Result and discussion}

It is observed that both sensor probes generate a comb like reflected spectrum as a broadband ASE is launched into the device. This is attributed to the optical path difference (OPD) between cladding and core modes, which produces an interference pattern in both the MZI and non-adiabatic tapered fibers. To reduce the influence of vibration on the sensor caused by air-flow, we used saturated salt to change the relative humidity in the testing chamber. The relative humidity in the chamber was varied between $10 \%$ and $80 \% \mathrm{RH}$ under a room temperature of $22^{\circ} \mathrm{C}$. At the start of experiment, we use silica gel into the chamber in order to decrease the humidity to $10 \%$. As the humidity level increased, the transmission spectrum of the probe was recorded by using the OSA. Fig. 5(a) and (b) show several measured spectra under different RH levels for the RH sensor configured with MZI and tapered fiber probes, respectively. It can be seen that resonant wavelengths of both probes red-shifted when the level of relative humidity increased. For instance, the resonant dip wavelength for MZI dumbbell shape increases from 1555.76 to $1556.34 \mathrm{~nm}$ as the RH increases from 10 to $80 \%$. While, for etched SMF the resonant dip wavelength increases from 1554.58 to $1554.85 \mathrm{~nm}$ as the $\mathrm{RH}$ increases from 10 to $80 \%$

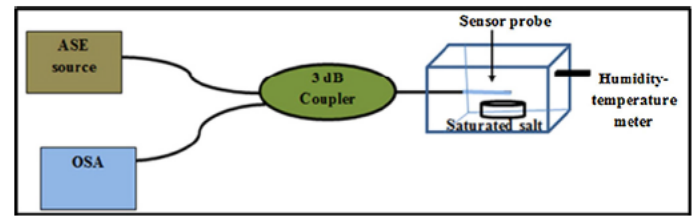

Fig. 4. The experimental setup for the proposed sensor to detect change in relative humidity using the fabricated inline dumbbell shaped MZI coated with HEC/PVDF composite.

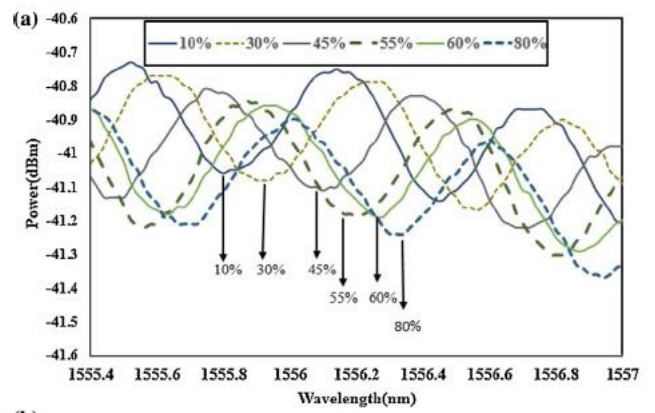

(b)

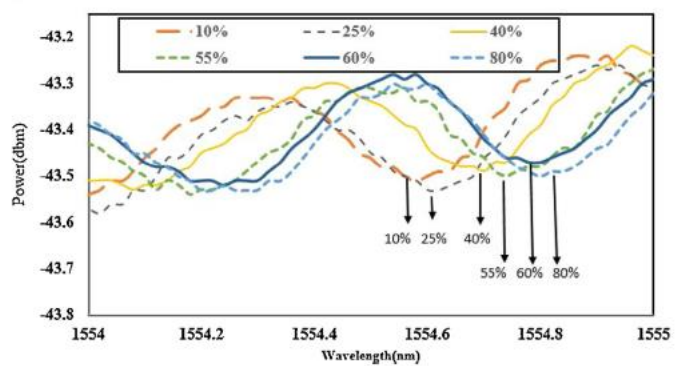

Fig. 5. (a) The variation of spectra under different RH levels for the RH sensor configured with MZI fiber probes. (b) The variation of spectra under different RH levels for the RH sensor configured with tapered fiber probes.

We use a combination of HEC and PVDF as a sensitive material for our probe. This material is relatively common and inexpensive. The mixture turns into a gel-like material after a long period of agitation, and finally stabilizes and forms into three-dimensional structure of the mesh gel after being coated onto the sensor probe. The mesh gel has a good absorption of water in the air. The response of the sensor to humidity change can be explained as follows. The HEC/PVDF composite in the form of mesh gel absorbs water molecules in its body. The absorbed water molecules increase the average density of the composite that results in an increase of its refractive index. The change of refractive index with different humidity or precipitation of moisture has good reproducibility. As a result, the resonant wavelengths of both sensors coated with the HEC/PVDF shift with RH level change. This is opposed to the trend demonstrated by the uncoated structure where its output spectrum remains constant despite the increase in relative humidity. Similar experimental results are expected if another peak wavelength is used as reference.

Fig. 6(a) and (b) shows the sensors response to humidity measured using resonant wavelength shift for both sensors. We find that the wavelength increases in a slight quadratic manner with the increase in $\mathrm{RH}$. The square regression coefficient values, which measure the goodness of fit, are 0.973 and 0.9574 for the dumbbell MZI and tapered SMF structures, respectively. The considerably high values of the coefficient allow the prediction of unknown relative humidity by the model.

Fig. 7 shows the linear relationship between humidity and resonant wavelength shift for both sensors. The sensors demonstrated shows a linear shift from 20 to $45 \%$. It is found that the MZI-based sensor has a sensitivity of $0.0123 \mathrm{~nm} / \%$ with a linearity of $99.88 \%$ and limit of detection of $0.44 \%$. On the other hand, the etched SMF structure also shows change in the resonant wavelength with the increase in $\mathrm{RH}$. The tapered fiber based sensor has a sensitivity of $0.0074 \mathrm{~nm} / \%$ with linearity of $98.85 \%$ and limit of detection of

Link to Full-Text Articles :

http://www.sciencedirect.com/science/article/pii/s0924424715000722

http://www.hindawi.com/journals/amse/2013/624314/abs/ 